$\begin{array}{ll}w_{0} & =\text { feed velocity by nozzle } \\ z & =\text { axial coordinate } \\ z_{0} & =\text { film thickness } \\ \beta & =\text { half angle at apex of cone } \\ \xi & =\text { dimensionless coordinate } \\ \xi_{0} & =\text { dimensionless film thickness } \\ \nu & =\text { kinematic viscosity of liquid } \\ \omega & =\text { angular velocity of disk }\end{array}$

\section{Literature Gited}

1) Bruin, S.: Chem. Eng. Sc., 24, 1647 (1969)

2) Emslie, A. G., F. T. Bonner and L. G. Peck: J. Appl. Phys., 29, 858 (1958)

3) Fraser, R. P., P. Eisenklam and N. Dombrowski: Brit. Chem.

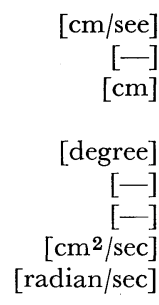

Eng., 2, 236 (1957)

4) Hinze, J. O. and H. Milborn: J. Appl. Phys., 17, 45 (1950)

5) Karman, Th.: Z. Angew. Math. Mech., 1, 233 (1921)

6) Marshall, W. R. and E. Selzer: Chem. Eng. Prog., 46, 501, 575 (1950)

7) Matsumoto, S., K. Saito and Y. Takashima: Bull. Tokyo Inst. Tech., No. 109, 69 (1972)

8) Matsumoto, S., K. Saito and Y. Takashima: ibid., No. 116, 85 (1973)

9) Miyasaka, Y.: Ph. D. Dissertation (Tohoku Univ.) (1969)

10) Nikolaev, V. S., K. D. Vachagin and Yu. N. Brryshev: Intern. Chem. Eng., 7, 595 (1967)

11) Oyama, Y. and K. Endou: Kagaku Kōgaku (Chèm. Eng., Japan), 17, 256 (1953)

12) Tanasawa, Y., Y. Miyasaka and M. Umehara: Trans. Soc. Mech. Engrs., 25, 897 (1958)

\title{
A STUDY OF MASS TRANSPORT IN TURBULENT FLOW*
}

\author{
Shiro ITO AND Kohei OGAWA \\ Department of Chemical Engineering, Tokyo Institute of \\ Technology, Tokyo, Japan
}

\begin{abstract}
Considering the analogical equation for mass transfer with the kinematic energy equation, a new eddy diffusivity is defined in much the same way as the eddy viscosity presented in the previous paper ${ }^{5}$. Being different from the conventional eddy diffusivity, the newly defined eddy diffusivity can be determined uniquely on the notice point from a single mean concentration gradient to any direction at that point.

At the transitional region from laminar to turbulent flow of liquid in a circular pipe, further, experimental values of the newly defined eddy diffusivity are obtained by simultaneous measurment of the liquid velocity fluctuations and the concentration fluctuations of injected tracer by electro-chemical techniques.
\end{abstract}

\section{Introduction}

The general equation of mass transfer for turbulent flow for constant density and the coefficient of molecular diffusion is obtained by substituting the concentration and velocity components, which are decomposed into their time-mean parts and fluctuating parts, into the general equation of mass transfer for nonturbulent flow and averaging with respect to time as follows (using Cartesian tensor notation).

$$
\frac{\partial \bar{C}}{\partial t}+\bar{U}_{i} \frac{\partial C_{e}}{\partial x_{i}}=\frac{\partial}{\partial x}\left(D \frac{\partial \bar{C}}{\partial x_{i}}-\overline{u_{i} c}\right)
$$

where $C$ denotes the concentration, $U_{i}$ is the velocity, $D$ is the coefficient of molecular diffusion, and the bars denote the time-mean parts and the small letters denote the fluctuating parts. Hereupon, the conventional eddy diffusivity, $\varepsilon_{c}$, has been defined analogically, with the

\footnotetext{
* Received on April 25, 1973

T152 東京都目黒区大岡山2-12-1

東京工業大学工学部化学工学科 小川浩平
}

definition of the conventional eddy viscosity as the following equation.

$$
-\overline{u_{i} c}=\varepsilon_{c}\left(\frac{\partial \bar{C}}{\partial x_{i}}\right)
$$

But this concept brings complexity when the abovedefined eddy diffusivity is applied to practical study of turbulent mixing phenomena. In a word, the plural values of the eddy diffusivity are decided at one notice point, and they complicate the analysis of mixing phenomena just as does the conventionally defined eddy viscosity.

It is thus the purpose of this paper to define a new eddy diffusivity which is determined uniquely on the notice point from a single mean concentration gradient. to any direction at that point, in much the same way as the definition of the eddy viscosity reported before ${ }^{5)}$.

\section{Theoretical Consideration}

Multiplication of mass transfer equation by $C$ and summation with respect to the index $i$ will yield after some transformation an equation which corresponds to 
the kinematic energy, as follows.

$$
\begin{aligned}
\frac{1}{2} \frac{\partial C^{2}}{\partial t}= & -\frac{1}{2} U_{i} \frac{\partial C^{2}}{\partial x_{i}}+D \frac{\partial}{\partial x_{i}}\left(C \frac{\partial C}{\partial x_{i}}\right) \\
& -D\left(\frac{\partial C}{\partial x_{i}}\right)\left(\frac{\partial C}{\partial x_{i}}\right)
\end{aligned}
$$

To express explicitly the contribution of the turbulent motion, each quantity is decomposed into its timemean part and its fluctuating part. Then substitution in Eq.(3) and averaging with respect to time give the corresponding equation of total kinematic energy.

$$
\begin{aligned}
\frac{1}{2} \frac{\partial \bar{C}^{2}}{\partial t}+\frac{1}{2} \frac{\partial c^{2}}{\partial t}= & -\frac{1}{2} \frac{\partial}{\partial x_{i}}\left(\bar{U}_{i} \bar{C}^{2}\right)-\frac{1}{2} \frac{\partial}{\partial x_{i}}\left(\bar{U}_{i} \bar{c}^{2}\right) \\
& +D \frac{\partial}{\partial x_{i}}\left(\bar{C} \frac{\partial \bar{C}}{\partial x_{i}}\right)+D \frac{\partial}{\partial x_{i}} \overline{\left(c \frac{\partial c}{\partial x_{i}}\right)} \\
& -D\left(\frac{\partial \bar{C}}{\partial x_{i}}\right)\left(\frac{\partial \bar{C}}{\partial x_{i}}\right)-D\left(\frac{\partial c}{\partial x_{i}}\right)\left(\frac{\partial c}{\partial x_{i}}\right) \\
& -\bar{C}\left(\overline{u_{i} \frac{\partial c}{\delta x_{i}}}\right)-\left(\overline{c u_{i}} \frac{\partial \bar{C}}{\partial x_{i}}\right)
\end{aligned}
$$

Multiplication by $\bar{C}$ of Eq.(1) gives the corresponding equation of kinematic energy for mean motion as follows.

$$
\begin{aligned}
\frac{1}{2} \frac{\partial \bar{C}^{2}}{\partial t}+\frac{1}{2} \frac{\partial}{\partial x_{i}}\left(\bar{U}_{i} \bar{C}^{2}\right)= & D \frac{\partial}{\partial x_{i}}\left(\bar{G} \frac{\partial \bar{C}}{\partial x_{i}}\right) \\
& -D\left(\frac{\partial \bar{C}}{\partial x_{i}}\right)\left(\frac{\partial \bar{C}}{\partial x_{i}}\right) \\
& -\bar{C}\left(\overline{u_{i} \frac{\partial c}{\partial x_{i}}}\right)
\end{aligned}
$$

Subtracting Eq.(5) from Eq.(4), the corresponding equation of kinematic energy for fluctuating motion is obtained.

$$
\begin{aligned}
\frac{1}{2} \frac{\partial \overline{c^{2}}}{\partial t}+\frac{1}{2} \frac{\partial}{\partial x_{i}}\left(\bar{U}_{i} \overline{c^{2}}\right)= & D \frac{\partial}{\partial x_{i}}\left(\overline{c \frac{\partial c}{\partial x_{i}}}\right) \\
& -D\left(\frac{\partial c}{\partial x_{i}}\right)\left(\frac{\partial c}{\partial x_{i}}\right)-\overline{c u_{i}} \frac{\partial \bar{C}}{\partial x_{i}}
\end{aligned}
$$

Hereupon, in the same manner as for the definition of the eddy viscosity ${ }^{5}$, the term equivalent to the energy dissipation term is singled out for investigation of the eddy diffusivity. The quantity corresponding to the dissipation energy per unit time of the mean motion, $D \Phi_{m c}$, is shown by the second term in the right-hand side of Eq.(5) as

$$
D \Phi_{m c}=D\left(\frac{\partial \bar{C}}{\partial x_{i}}\right)\left(\frac{\partial \bar{C}}{\partial x_{i}}\right)
$$

The other side, the quantity corresponding to the dissipation energy per unit time of the fluctuating motion, $D \Phi_{f c}$, is shown by the second term in the right-hand side of Eq.(6) as

$$
D \Phi_{f c}=D \overline{\left(\frac{\partial c}{\partial x_{i}}\right)\left(\frac{\partial c}{\partial x_{i}}\right)}
$$

Hence, it is assumed that the above quantity corre- sponding to the dissipation energy of the fluctuating motion is $k_{c}$ times of that of the mean motion, as follows.

$$
D \Phi_{f c}=k_{c} D \Phi_{m c}
$$

Accordingly, the quantity which corresponds to the total dissipation energy per unit time, $D \Phi_{t c}$, can be shown as

$$
\begin{aligned}
D \Phi_{t c} & =D \Phi_{m c}+D \Phi_{f c} \\
& =\left(1+k_{c}\right) D \Phi_{m c}
\end{aligned}
$$

Eq.(10) shows that the quantity corresponding to the total dissipation energy in turbulent flow is $\left(1+k_{c}\right)$ times the quantity corresponding to the dissipation energy of the mean motion in consequence of the disturbances. In other words, it can be said that the apparent diffusivity in turbulent flow can be shown as $\left(1+k_{c}\right) D$. Therefore, multiplication by $D$ of the coefficient $k_{c}$ will be regarded as the eddy diffusivity, $\varepsilon_{c 0}$, as follows.

$$
\varepsilon_{c 0}=k_{c} D=\frac{D \Phi_{f c}}{D \Phi_{m c}} D=\frac{\overline{\left(\frac{\partial c}{\partial x_{i}}\right)\left(\frac{\partial c}{\partial x_{i}}\right)}}{\left(\frac{\partial \bar{C}}{\partial x_{i}}\right)\left(\frac{\partial \bar{C}}{\partial x_{i}}\right)} D
$$

The above newly defined eddy diffusivity is a scalar value determined uniquely at the notice point from a single mean concentration gradient to any direction at the point.

Values of the above-defined eddy diffusivity can be obtained easily if the concentration fluctuation gradient to each direction is known. But at present it is generally difficult to make an adequately small probe for measuring the concentration fluctuation gradient to each direction. However, it is possible to know the values of the above-defined eddy diffusivity through relating this eddy diffusivity and the mean concentration gradient by applying the representative mixing time for mass transfer as follows.

Turbulent flow is looked upon as a field in which turbulent lumps move transversely to the mean field. The quantity equivalent to the kinematic energy of the turbulent lumps per unit mass is shown as $\overline{c^{2}} / 2$, and the quantity corresponding to the dissipation energy losses occurring in the time $\Delta t_{c}$ can be shown as $D \Phi_{f c} \Delta t_{c}$. Assuming that the above quantity, $\overline{c^{2}} / 2$, is consumed by the in time $\Delta t_{c}$, the following relation can be considered.

$$
\frac{1}{2} \overline{c^{2}}=D \Phi_{f c} \cdot \Delta t_{c}
$$

Considering that negative acceleration acts upon the moving turbulent lumps, further, the quantity $\sqrt{\overline{c^{2}}}$, which corresponds to the representative velocity, becomes zero in time $\Delta t_{c}$ by the action of this effect. Assuming that this effect can be shown with the product of the representative velocity, $\sqrt{\overline{q^{2}}}$, and the representative mean concentration gradient, $\sqrt{\Phi_{m c}}$, the following relative equation can be obtained. 


$$
\sqrt{\overline{c^{2}}}-\sqrt{\overline{q^{2}}} \cdot \sqrt{\Phi_{m c}} \cdot \Delta t_{c}=0
$$

Then the representative mixing time $\Delta t_{c}$ can be written as

$$
\Delta t_{c}=\frac{\sqrt{\overline{c^{2}}}}{\sqrt{\overline{q^{2}} \cdot \sqrt{\Phi_{m c}}}}
$$

Substituting Eq.(14):into Eq.(12) gives

$$
\frac{1}{2} \bar{c}^{2}=D \Phi_{f c} \cdot \frac{\sqrt{\overline{c^{2}}}}{\sqrt{\bar{q}^{2}} \cdot \sqrt{\overline{\Phi_{m c}}}}
$$

From Eq.(15) and Eq.(9) the newly defined eddy diffusivity can be shown as

$$
\varepsilon_{c 0}=k_{c} D=\frac{\sqrt{\overline{\overline{q^{2}}}} \cdot \sqrt{\overline{c^{2}}}}{2\left\{\left(\frac{\partial \overline{C_{i}}}{\partial x_{i}}\right)\left(\frac{\partial \overline{C_{i}}}{\partial x_{i}}\right)\right\}^{1 / 2}}
$$

It is possible to know the values of $\sqrt{\overline{q^{2}}}, \sqrt{\overline{c^{2}}}$ and $\sqrt{\Phi_{m c}}$ with present measuring techniques.

It can be considered that the turbulent lumps receive negative acceleration, $-\sqrt{\overline{q^{2}}} \sqrt{\Phi_{m}}$, in moving transversely to the mean field, as reported before ${ }^{5)}$. Therefore the moving distance of the turbulent lumps in time $\Delta t_{c}$ can be introduced as follows.

$$
\begin{aligned}
L_{c} & =\sqrt{\overline{q^{2}}} \frac{\sqrt{\overline{c^{2}}}}{\sqrt{\overline{q^{2}}} \sqrt{\Phi_{m c}}}-\frac{1}{2} \sqrt{\overline{q^{2}}} \sqrt{\Phi_{m}} \frac{\overline{c^{2}}}{\overline{q^{2}} \Phi_{m c}} \\
& =\frac{\sqrt{c^{2}}}{\sqrt{\overline{\Phi_{m c}}}}\left(1-\frac{1}{2} \frac{\sqrt{\overline{c^{2}}} \sqrt{\Phi_{m}}}{\sqrt{\overline{q^{2}}} \sqrt{\Phi_{m c}}}\right)
\end{aligned}
$$

where $\sqrt{\Phi_{m}}$ denotes the representative mean velocity gradient as

$$
\sqrt{\Phi_{m}}=\left\{\left(\frac{\partial U_{i}}{\partial x_{j}}+\frac{\partial U_{j}}{\partial x_{i}}\right) \frac{\partial U_{i}}{\partial x_{j}}\right\}^{1 / 2}
$$

The above newly defined eddy diffusivity and the representative moving distance of the turbulent lumps are determined uniquely at the notice point by a single mean concentration and mean velocity gradient to any direction at the point.

Eq.(16) can be written in cylindrical coordinates as

$$
\varepsilon_{c 0}=\sqrt{\overline{q^{2}}} \sqrt{\overline{c^{2}}} / 2\left\{\left(\frac{\partial \bar{C}}{\partial r}\right)^{2}+\left(\frac{1}{r} \frac{\partial \bar{C}}{\partial \theta}\right)^{2}+\left(\frac{\partial \bar{C}}{\partial z}\right)^{2}\right\}^{1 / 2}
$$

In the case of the diffusion of solution into liquid flowing through a circular pipe, the above-defined eddy diffusivity can be written as follows when $\partial / \partial \theta=0$ and $\partial / \partial z \ll \partial / \partial r$

$$
\varepsilon_{c 0}=\frac{\sqrt{\overline{q^{2}}} \sqrt{\overline{c^{2}}}}{2\left|\frac{\partial \bar{C}}{\partial r}\right|}
$$

The shape of the above-defined eddy diffusivity is similar to the conventional definition as shown in Eq. (2).

In the case of a tracer injected at the pipe center, the values of above $\varepsilon_{c 0}$ at the pipe center cannot obtained, for $\partial \bar{C} / \partial r$ takes zero value at that position, but it is possible to estimate the values with other values at positions near the pipe center.
Subsequently, the turbulent Schmidt number, $S c_{t}$, which is used frequently as an index of the character of turbulent mass transport, is considered. This dimensionless number can be shown as follows by making use of the above parameters.

$$
S_{c_{t}}=\frac{k_{\nu}}{k_{c} D}=\frac{k}{k_{c}} S c
$$

where $k_{\nu}$ is the newly-defined eddy viscosity in the previous paper $^{5)}$.

$$
k_{\nu}=\frac{\overline{q^{2}}}{2 \sqrt{\Phi_{m}}}
$$

In the particular case where the above $S c_{t}$ takes unit value the representative mixing time of mass transfer, $\Delta t_{c}$, and that of momentum transfer, $\Delta t$, become

$$
\Delta t_{c}=\frac{\sqrt{\overline{c^{2}}}}{\sqrt{\overline{q^{2}}} \sqrt{\overline{\Phi_{m c}}}}=\Delta t=\frac{1}{\sqrt{\Phi_{m}}}
$$

and the representative moving distance for mass transfer, $L_{c}$, and that for momentum transfer, $L$, become

$$
L_{c}=\frac{\sqrt{\overline{c^{2}}}}{2 \cdot \sqrt{\Phi_{m c}}}=L=\frac{\sqrt{\overline{q^{2}}}}{2 \sqrt{\Phi_{m}}}
$$

Accordingly, it is clear that the representative mixing time and length of mass transport is the same as that of momentum transfer when the turbulent Schmidt number takes unit value.

\section{Experiments}

In the laminar-turbulent transitional region of liquid flow in a circular pipe the newly defined eddy diffusivity in conformity with Eq.(19) is calculated by simultaneous measuring of the velocity fluctuations and the concentration fluctuations with electro-chemical techniques of a tracer injected at the pipe center.

\section{Simultaneous measuring method of velocity}

\section{fluctuations and concentration fluctuations}

For measuring liquid velocity fluctuations and concentration fluctuations at the same time, the recent liquid velocity measuring method, based on electrochemical reaction controlled by diffusional mass transfer rate, is combined with the ordinary concentrationmeasuring method based on electro-conductivity.

Considering that the most-used electrolytic reaction $^{2,3)}$ for measuring liquid velocity is the oxidation of ferrocyanide ion on a platinum electrode in an electrolyte which contains a large amount of potassium chloride as the supporting electrolyte,

$$
\mathrm{Fe}(\mathrm{CN})_{6}^{4-} \longrightarrow \mathrm{Fe}(\mathrm{CN})_{6}^{3-}+e
$$

potassium chloride is considered to be the most suitable tracer for measuring the concentration fluctuations.

With gradual addition of an aqueous solution of 1 mole/l potassium chloride to an aqueous solution which contains $3 \times 10^{-3}$ mole $/ l$ potassium ferrocyanide and potassium ferricyanide, the effect of the potassium chloride concentration on the velocity-measuring 


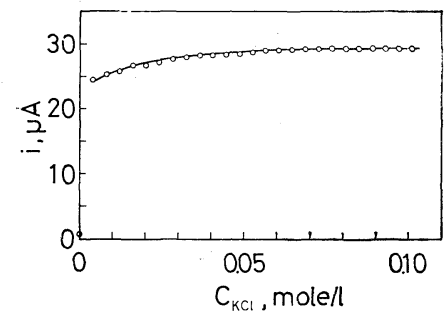

Fig. 1 Effect of $\mathrm{KCl}$ concentration on diffusional curreut

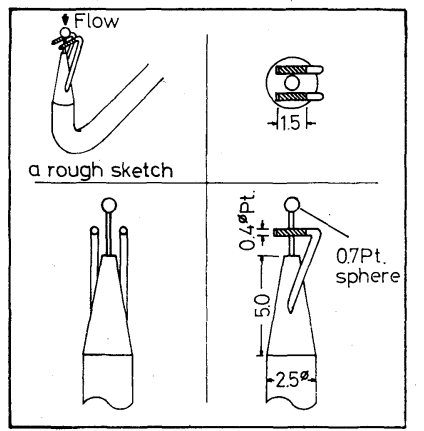

Fig. 2 Probe for measuring velocity and concentration at the same time

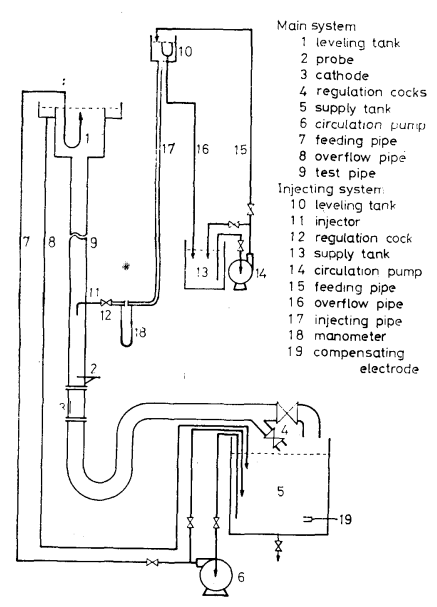

Fig. 3 Schematic diagram of apparatus

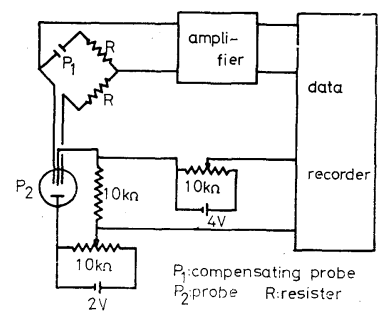

Fig. 4 Electric circuit

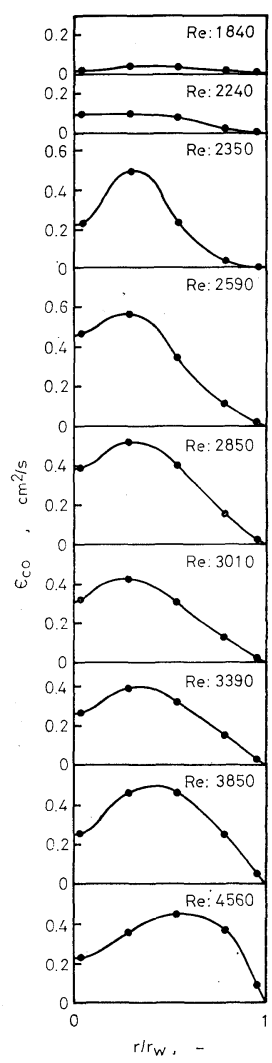

Fig. 5 Eddy diffusivity method is examined experimentally. Consequently, when potassium chloride concentration is more than $0.08 \mathrm{~mole} / l$ the diffusion current is not affected by more added potassium chloride, as shown in Fig. 1. Therefore, if the potassium chloride concentration of the main flow is set as 0.1 mole $/ l$, it is possible to measure the velocity fluctuations and concentration fluctuations of an injected tracer which has a concentration greater than that of the main flow at the same time.

The probe shown in Fig. $\mathbf{2}$ is designed to measure the velocity fluctuations and the injecting tracer concentration fluctuations in circular-pipe flow. That is the concentration fluctuations can be measured at the fully mixing zone downstream of the velocity-measuring sphere probe.

It was confirmed that a platinum sphere can be used as a probe to measure the time-mean values of the square of energy of turbulence, $\overline{q^{2}}$, in circular-pipe flow by investigation of the angular characteristic of this type of probe $^{5)}$.

It was confirmed that chemical reaction is occurring at the surface of the upstream velocity-measuring probe has no effect upon the downstream electro-conductivity measuring.

\section{Experimental apparatus and method}

The experimental apparatus is illustrated schematically in Fig. 3. In the main system, the liquid in a storage tank is fed into a levelling tank with a pump and flows down through the test pipe connected at the bottom of the levelling tank and out of the system. The overflow liquid from the levelling tank flows back to the storage tank directly. A test pipe of $48 \mathrm{~mm}$ inner diameter, $6.5 \mathrm{~m}$ total length and $1.665 \times 10^{-6}$ relative roughness is set up vertically. The test position where the measuring probe is mounted is set $5.3 \mathrm{~m}$ from the test pipe inlet.

The opposite electrode for velocity measurement, made of thin platinum sheet, is attached to the pipe inner wall downstream of the measuring probe. The rate of flow is regulated by two regulation cocks connected to the test pipe.

In the tracer injecting system, the potassium chloride solution for the tracer in the storage tank is fed into the levelling tank with a pump, flows through the pipe connected to the bottom of the levelling tank and is injected into the main test pipe at an arbitrary point upstream of the measuring probe. The overflow tracer liquid from the levelling tank flows back to the storage tank directly.

An L-shaped injection pipe of $1.5 \mathrm{~mm}$ inner diameter is set up vertically at the center of the main test pipe. The flow rate of tracer solution is regulated as the same mean velocity of the main flow at the center of the test pipe by a regulation cock connected to the injection pipe.

The material of most of the apparatus is PVG plastics, and the liquid does not touch metal except the probe and the injector tip.

The electrolyte is composed of $3 \times 10^{--3}$ mole $/ l$ potassium ferrocyanide and potassium ferricyanide and 0.1 mole $/ l$ potassium chloride as the supporting electrolyte. And it was confirmed by experiment that the 
kinematic viscosity of this electrolyte has the same values as that of water ${ }^{4}$.

The concentration of the potassium chloride solution for the tracer is 1.0 mole $/ l$, and the set position of the tracer injection is changed from $3 \mathrm{~cm}$ to $80 \mathrm{~cm}$ upstream of the measuring probe.

The measuring probe can be traversed in a radial direction at that test section, and the data are obtained at five radii $\left(0.96 r_{w}, 0.79 r_{w}, 0.54 r_{w}, 0.29 r_{w}, 0.04 r_{w}\right.$; $r_{w}$ is the pipe inner radius) at any flow rate.

The electrical circuit diagram is shown in Fig. 4.

The signals recorded on magnetic tape are converted to digital quantities and treated statistically by a computer.

\section{Experimental results}

The radial distribution of the newly defined eddy diffusivity in conformity with Eq.(19) is shown in Fig. 5 at several Reynolds numbers. Now, the data supplied for computation is that at a position where the assumption of $\partial \bar{C} / \partial r \gg \partial \bar{C} / \partial z \doteqdot 0$ is satisfied. At low Reynolds numbers (900-1800), the values of the eddy diffusivity are negligibly small at any radial position. With the increasing Reynolds number values come to take a significant trend.

At Reynolds numbers (1800-2200) at which no highfrequency velocity fluctuations exist at any radial position, the values of eddy diffusivity are small and the radial distribution curve of the eddy diffusivity is almost flat. With further increase of Reynolds number, the values of this eddy diffusivity at the intermediate part of the pipe center and the pipe wall are larger than that of other radii, and the values of this eddy diffusivity become extremely large at Reynolds number (2300-2400) at which high-frequency velocity fluctuations can be noticed in the larger-amplitude disturbances. As the larger-amplitude disturbances decrease, the pipe sectional mean values of this eddy diffusivity become small, and near Reynolds number 3400 this value becomes minimum. This Reynolds number coincides with the upper limit of the Reynolds number in the laminar-turbulent transitional region ${ }^{3)}$. With further increase of Reynolds number, the peak of the distribution curve moves gradually towards the pipe wall. And at Reynolds numbers above 4560, the shape of radial distribution curves of the eddy diffusivity does not change.

\section{Gonclusion}

The new eddy diffusivity is defined analogically with the definition of the eddy viscosity. This newly defined eddy diffusivity is settled uniquely on the notice point by a single mean concentration gradient to any direction at that point. Introducing the consideration that the turbulent field can be looked upon as a flow field in which turbulent lumps move transversely to the mean field, the representative mixing time is defined. It is clear that mass transport can be seen is the same as momentum where the turbulent Schmidt number takes unit value by the examination of the representative mixing time and the moving distance of the turbulent lumps.

In the laminar-turbulent transitional region of liquid flow in a circular pipe, experimental values of the newly defined eddy diffusivity are obtained by measuring the liquid velocity fluctuations and the concentration fluctuations of a tracer injected into the pipe center at the same time.

\section{Nomenclature}

\begin{tabular}{|c|c|c|c|}
\hline$C$ & $=$ & concentration & ole $\left./ \mathrm{cm}^{3}\right]$ \\
\hline $\bar{C}$ & $=$ & time-mean concentration & $\left.\mathrm{ole} / \mathrm{cm}^{3}\right]$ \\
\hline$c$ & $=$ & concentration fluctuation & ole $\left./ \mathrm{cm}^{3}\right]$ \\
\hline$D$ & $=$ & coefficient of molecular diffusion & {$\left[\mathrm{cm}^{2} / \mathrm{s}\right]$} \\
\hline$k$ & $=$ & coefficient about turbulent dissipation energy & {$[-]$} \\
\hline$k_{c}$ & $=$ & $\begin{array}{l}\text { coefficient about the quantity which } \\
\text { corresponds to dissipation energy }\end{array}$ & {$[-]$} \\
\hline$L$ & $=$ & $\begin{array}{l}\text { representative mixing length for } \\
\text { momentum transfer }\end{array}$ & {$[\mathrm{cm}]$} \\
\hline$L_{c}$ & $=$ & $\begin{array}{l}\text { representative moving distance of turbulent } \\
\text { elements for mass transfer }\end{array}$ & {$[\mathrm{cm}]$} \\
\hline$q^{2}$ & $=$ & $u_{i} u_{i}$, square of energy of turbulence & {$\left[\mathrm{cm}^{2} / \mathrm{s}^{2}\right]$} \\
\hline$R e$ & $=$ & $2<\bar{U}_{z}>r_{w} / \nu$, Reynolds number & {$[-]$} \\
\hline$r$ & $=$ & radius & {$[\mathrm{cm}]$} \\
\hline$r_{w}$ & $=$ & pipe inner radius & {$[\mathrm{cm}]$} \\
\hline$t$ & $=$ & time & {$[\mathrm{s}]$} \\
\hline$\underline{U_{i}}$ & $=$ & velocity & {$[\mathrm{cm} / \mathrm{s}]$} \\
\hline $\bar{U}_{i}$ & $=$ & time-mean velocity & {$[\mathrm{cm} / \mathrm{s}]$} \\
\hline & $=$ & velocity fluctuation & {$[\mathrm{cm} / \mathrm{s}]$} \\
\hline$<\bar{U}_{z}>$ & $=$ & cross-sectional average velocity & {$[\mathrm{cm} / \mathrm{s}]$} \\
\hline$\Delta t$ & $=$ & $\begin{array}{l}\text { representative mixing time of momentum } \\
\text { transfer }\end{array}$ & s] \\
\hline$\Delta t_{c}$ & $=$ & representative mixing time of mass transfer & [s] \\
\hline$\varepsilon_{c}$ & $=$ & conventional eddy diffusivity & {$\left[\mathrm{cm}^{2} / \mathrm{s}\right]$} \\
\hline$\varepsilon_{c 0}$ & $=$ & eddy diffusivity proposed in this paper & {$\left[\mathrm{cm}^{2} / \mathrm{s}\right]$} \\
\hline$\nu$ & $=$ & kinematic eddy viscosity & {$\left[\mathrm{cm}^{2} / \mathrm{s}\right]$} \\
\hline
\end{tabular}

\section{Literature Cited}

1) Hinze, J. O.: “Turbulence", McGraw-Hill (1959)

2) Ranz, W. E.: AIChE J., 4, 338 (1958)

3) Ito, S., K. Ogawa and S. Urushiyama: J. Chem. Eng. Japan, 4, $128(1971)$

4) Ito, S., K. Ogawa and T. Yuhara: Kagaku Kögaku, 37, 698 (1973)

5) Ito, S. and K. Ogawa: J. Chem. Eng. Japan, 6, 231 (1973) 